\title{
Advanced backcross QTL mapping of resistance to Fusarium head blight and plant morphological traits in a Triticum macha $\times T$. aestivum population
}

\author{
Maria Buerstmayr • Marc Lemmens • \\ Barbara Steiner $\cdot$ Hermann Buerstmayr
}

Received: 13 January 2011 / Accepted: 23 March 2011/Published online: 11 April 2011

(C) The Author(s) 2011. This article is published with open access at Springerlink.com

\begin{abstract}
While many reports on genetic analysis of Fusarium head blight (FHB) resistance in bread wheat have been published during the past decade, only limited information is available on FHB resistance derived from wheat relatives. In this contribution, we report on the genetic analysis of FHB resistance derived from Triticum macha (Georgian spelt wheat). As the origin of T. macha is in the Caucasian region, it is supposed that its FHB resistance differs from other well-investigated resistance sources. To introduce valuable alleles from the landrace T. macha into a modern genetic background, we adopted an advanced backcross QTL mapping scheme. A backcrossderived recombinant-inbred line population of $321 \mathrm{BC}_{2} \mathrm{~F}_{3}$ lines was developed from a cross of T. macha with the Austrian winter wheat cultivar Furore. The population was evaluated for Fusarium resistance in seven field experiments during four seasons using artificial inoculations. A total of 300 lines of the population were genetically fingerprinted using SSR and AFLP markers. The resulting linkage map covered 33 linkage groups with 560 markers. Five novel FHB-resistance QTL, all descending from T. macha, were found on four chromosomes (2A, 2B, 5A, 5B). Several QTL for morphological and developmental traits were mapped in the same population, which partly overlapped with FHB-resistance QTL. Only the 2BL FHBresistance QTL co-located with a plant height QTL. The largest-effect FHB-resistance QTL in this population
\end{abstract}

Communicated by C. Feuillet.

M. Buerstmayr · M. Lemmens · B. Steiner · H. Buerstmayr ( $\square)$ Department for Agrobiotechnology Tulln, BOKU,

University of Natural Resources and Life Sciences Vienna,

Konrad Lorenz Str. 20, 3430 Tulln, Austria

e-mail: hermann.buerstmayr@boku.ac.at mapped at the spelt-type locus on chromosome 5A and was associated with the wild-type allele $q$, but it is unclear whether $q$ has a pleiotropic effect on FHB resistance or is closely linked to a nearby resistance QTL.

\section{Introduction}

Resistance to Fusarium head blight (FHB) is one of the most important traits for modern wheat varieties in many wheat growing areas worldwide. Resistance to FHB is a quantitative trait, governed by polygenes, and quantitative trait loci have been detected on all wheat chromosomes (Buerstmayr et al. 2009; Liu et al. 2009; Löffler et al. 2009). Apart from active physiological resistance plant developmental and morphological characters, especially plant height, flowering time, spike morphology and environmental conditions modulate disease development. The complex nature of the resistance and the important role of genotype-by-environment interactions render breeding for improved FHB resistance difficult. Large genetic variation for FHB resistance is available in the wheat gene pool, but often the regionally best adapted and most highly productive cultivars are susceptible to FHB (Buerstmayr et al. 2009).

Considering the resistance sources used for genetic analysis thus far, two main approaches can be distinguished. One is to evaluate and map populations based on agronomically adapted cultivars, with moderate to good FHB resistance. The other is to employ and genetically characterize more or less exotic resistance sources, such as introduced cultivars, landraces or alien species. Unadapted or exotic genotypes are usually agronomically inferior to modern varieties. In populations from bi-parental crosses between an adapted parent and an exotic parent, the desired 
resistance traits may be confounded with 'wild' plant traits such as excessive height or spike morphology. To allow target traits from exotic parents to be evaluated in a more adapted genetic background, the advanced backcross quantitative trait locus (AB-QTL) scheme was proposed by Tanksley and Nelson (1996) for combining QTL detection with variety development. Populations are generated by repeated backcrossing to an adapted elite parent. In such backcross-derived lines, donor chromosome fragments are distributed throughout the genome in a standardized genetic background close to the elite parent. Repeated backcrossing increases recombination events between the remaining donor genome and elite genome, which leads to smaller donor fragments and enhances chances of separating linked genes. Molecular marker techniques and QTL mapping routines adjusted to this specific population design will reveal favourable alleles. Several AB-QTL analyses of different wheat populations identified valuable QTL alleles derived from exotic donor lines (Huang et al. 2004; Kunert et al. 2007; Leonova et al. 2007; Liu et al. 2006; Narasimhamoorthy et al. 2006; Naz et al. 2008).

In the present work, an advanced backcross population derived from a cross between a well-adapted Austrian bread wheat cultivar and Triticum macha as donor was screened for FHB-resistance QTL. T. macha, a hulled hexaploid wheat, is endemic to the Caucasus area where it was discovered in 1929. In Georgia, a popular local variety 'Makha' that had stable yields in different climatic conditions and high resistance to various diseases was grown until the 1930s (Barisashvili and Gorgidze 1979). Previous work identified a line of $T$. macha with remarkably high level of quantitative FHB resistance almost comparable to that of Sumai-3 (Buerstmayr et al. 1996; Grausgruber et al. 1998; Mentewab et al. 2000). This resistant line was used as donor parent. Investigations of Cao et al. (2000) confirm the phylogenetic difference between T. macha and common wheat as well as between T. macha and T. spelta. Because of the distinct origin of T. macha, we considered it likely that T. macha harbours novel resistance QTL.

Plant morphology, especially spike-related traits, differs considerably between the parents of the investigated population. Gross morphology of wheat spike is substantially influenced by the three major genes: $Q$ (speltoid ear, Faris et al. 2005; Mac Key 1954), $C$ (compact ear, Rao 1972) and S1 (sphaerococcum grains, Rao 1977; Salina et al. 2000). T. aestivum wheats carry the alleles $Q Q$ cc S1S1, and T. macha possesses $q q$ CC S1S1 (Morris and Sears 1967; Swaminathan and Rao 1961). A population from T. aestivum $\times T$. macha is therefore expected to segregate at $Q / q$ (square-headed and free threshing/speltoid and nonfree threshing, chromosome 5A) and $C / c$ (compact spike/ non-compact spike, chromosome 2D), but should be fixed at $S 1$ (non-sphaerococcum grains, chromosome 3D). Plant height (Buerstmayr et al. 2000; Gervais et al. 2003; Hilton et al. 1999; Mesterhazy 1995; Steiner et al. 2004), compactness of the spikes (Steiner et al. 2004; Zhu et al. 1999), flowering time (Gervais et al. 2003; Holzapfel et al. 2008; Paillard et al. 2004; Schmolke et al. 2005; Steiner et al. 2004) and flower opening (Gilsinger et al. 2005) have been reported as associated with FHB infection and development. Therefore, in this study, special attention was given to morphological traits and their association with FHB resistance.

A previous study with T. macha showed that its FHB resistance is located on several wheat chromosomes (Grausgruber et al. 1998; Mentewab et al. 2000). To date, only one T. macha-derived FHB-resistance QTL at chromosome 4A was mapped, in a single chromosome recombinant population (Steed et al. 2005).

We report here about an $\mathrm{AB}-\mathrm{QTL}$ analysis where a $\mathrm{BC}_{2}-$ derived population was used for simultaneous map calculation and genome-wide QTL detection. The aim of this study was to genetically characterize novel QTL for FHB resistance derived from $T$. macha and for morphological traits and to investigate their associations.

\section{Materials and methods}

Plant material and population development

A single homozygous line of T. macha was crossed to T. aestivum cultivar Furore, and $\mathrm{F}_{1}$ plants were backcrossed as maternal plants to Furore. A total of 321 independent $\mathrm{BC}_{1} \mathrm{~F}_{1}$ plants were backcrossed as female to Furore. From each of the 321 individually obtained $\mathrm{BC}_{2} \mathrm{~F}_{1}$ plants, one $\mathrm{BC}_{2} \mathrm{~F}_{3}$ plant was derived by single-seed descent. Bulked seed from each $\mathrm{BC}_{2} \mathrm{~F}_{3}$ plant was propagated in $0.5 \mathrm{~m}^{2}$ $\mathrm{BC}_{2} \mathrm{~F}_{3: 4}$ plots. The resulting seed was used for multienvironment resistance evaluations as $\mathrm{BC}_{2} \mathrm{~F}_{3: 5}$ lines. The recurrent parent Furore is an Austrian winter wheat cultivar with pedigree POKAL//HP35719/EXTREM/3/CAROLUS/ P9094. Furore is moderately susceptible to Fusarium head blight. The donor parent T. macha is a Transcaucasian landrace. It is a hexaploid hulled wheat with brittle rachis, has a dense but non-compact spike phenotype and pubescent and glaucous glumes. The T. macha line used in this project was originally obtained from JIC Norwich, UK (JIC accession 1240001), which was the same accession used for the generation of the T. macha-Hobbit-'sib' single chromosome substitution lines described by Law and Worland (1996) and analysed for FHB resistance by Grausgruber et al. (1998) and Mentewab et al. (2000). It showed excellent resistance to FHB in previous investigations (Buerstmayr et al. 1996). 
Fusarium head blight resistance evaluation

\section{Field design}

The $321 \mathrm{BC}_{2} \mathrm{~F}_{3: 5}$ lines, their parental lines and several control lines were evaluated in seven field experiments from 2005 to 2008 at the IFA-Tulln experiment farm, $30 \mathrm{~km}$ west of Vienna $\left(16^{\circ} 04^{\prime} \mathrm{E}, 48^{\circ} 19^{\prime} \mathrm{N}, 177 \mathrm{~m}\right.$ above sea level).

Each experiment was defined by a Fusarium isolate: $F$. culmorum $(\mathrm{FC})$ or $F$. graminearum $(\mathrm{FG})$ and a specific year encoded by the unit and the decade. For example, the experiment FC06 was the F. culmorum-inoculated experiment conducted in 2006. Each experiment had a randomized complete block design with two replications. The plot size was a double row of $0.8 \mathrm{~m}$ length with $17 \mathrm{~cm}$ row spacing. Sowing time was late autumn (October to November) in all years. The two blocks in each experiment were sown 2-3 weeks apart depending on the specific weather conditions, which delayed anthesis by 1-3 days for the later-sown blocks. Seed treatment, sowing density and crop management were essentially as described by Buerstmayr et al. (2002).

\section{Disease inoculation}

For six experiments, spray inoculation and for one experiment (FG08) grain spawn inoculation methods were applied. For spray inoculation, two different Fusarium isolates were used, either macroconidia of the $F$. culmorum single-spore isolate 'IFA-106' prepared as described by Buerstmayr et al. (2000) or the $F$. graminearum singlespore isolate 'IFA-65' prepared as described by Buerstmayr et al. (2002). We used a highly aggressive $F$. culmorum isolate and a moderately aggressive $F$. graminearum isolate. Conidia stock solutions in distilled water $(500 \times)$ were aliquoted into plastic vials and stored at $-30^{\circ} \mathrm{C}$ until use. At each inoculation day, the needed number of inoculum aliquots were thawed and diluted in deionized water to the desired concentration of $2.5 \times 10^{4}$ for the $F$. culmorum isolate and to $5 \times 10^{4}$ for $F$. graminearum isolate. Each plot was individually inoculated twice, first when $50 \%$ of the plants had reached anthesis and again 2 days later. Using a battery-driven backpack sprayer, inoculum was sprayed onto the heads until runoff. Inoculations were carried out in the evenings, usually from 4 to 6 p.m. An automated mist-irrigation system, triggered by leaf wetness measurement, maintained humidity and kept the plants wet for $20 \mathrm{~h}$ after inoculation. For grain spawn inoculation, $15 \mathrm{~g} \mathrm{~m}^{-2}$ Gibberella zeae-infected scabby maize grains were spread throughout the experiment 5 weeks before anthesis. The infested grains were taken from a maize field experiment, which was inoculated with a mix of three
F. graminearum (teleomorph: Gibberella zeae) isolates in the previous season. Also, this trial was mist irrigated using the same time schedule as described above during the flowering period to provide optimal infection conditions.

\section{Assessment of disease severity}

FHB severity was recorded on days 10, 14, 18, 22 and 26 after the first inoculation or after $50 \%$ anthesis for the spray and the grain spawn-inoculated experiments, respectively. Severity was estimated as the percentage of infected spikelets per plot by visually averaging whole plots.

\section{Evaluation of other traits}

All lines were evaluated for date of anthesis, plant height, spike length, number of spikelets per spike, spike density, threshability, spike glaucousness, hairiness of glumes and severity of leaf chlorosis. As an index of earliness, the date of 50\% anthesis was recorded for each plot and converted into the number of days after May 1. Plant height was measured as the distance in centimetre from the soil surface to the top of the ears excluding awns in the experiments FG05, FG06, FG07, FC07 and FG08. Spike length in centimetre and number of spikelets per spike were determined on six randomly chosen ears per plot from a noninoculated field experiment in 2009. The average number of spikelets per centimetre was calculated and used as measurement for spike density. Plots from this non-inoculated trial were harvested and threshed with a combine harvester (Nursery Master, Wintersteiger, Ried, Austria). Percent threshability was calculated as the weight proportion of free threshed seeds in all seeds harvested. In the experiments, FC07, FG07, FC08 and FG08 verdant spikes were assessed visually for glaucousness based on a 0 (nonglaucous) to 9 (intensely glaucous) scale. Similarly, the severity of physiological leaf chlorosis was scored on a 0 (non-chlorotic) to 9 (severely chlorotic) scale in experiments FG05, FG06, FC06, FG07, FC07 and FG08 at the heading stage. In addition, every line was classified for possessing hairy glumes, non-hairy glumes or heterogeneity for this trait. This trait was treated as a morphological marker.

\section{Molecular marker analysis}

Of 321 lines, 300 were randomly chosen for marker analysis. Genomic DNA was extracted from young leaves based on the CTAB method (Saghai Maroof et al. 1984) from ten pooled plants of each $\mathrm{BC}_{2} \mathrm{~F}_{3: 4}$ line and the parental lines. For molecular genotyping, microsatellite (simple sequence repeat, SSR) and amplified fragment length polymorphism (AFLP) markers were applied. 


\section{SSR marker analysis}

SSR marker analysis was done as described by Steiner et al. (2004) by fluorescent detection of PCR fragments on an LI-COR 4200 dual-dye DNA analyser (LI-COR Biosciences, Lincoln, Nebraska USA). The parents were checked for polymorphism with 282 SSR primer pairs, of which 128 polymorphic pairs were chosen for genotyping 300 lines of the mapping population, composed of 89 GWM markers (Roeder et al. 1998), 27 BARC markers (Song et al. 2005), 9 WMC markers (Somers et al. 2004), 1 GDM marker (Pestsova et al. 2000) and 2 markers within the $Q$ locus. For these, we compared the reported genome sequence of T. aestivum, GenBank no AY702956 ( $Q$ allele), and T. macha, GenBank no AY714342 ( $q$ allele), and designed two primer pairs flanking the variable microsatellites CCT repeat within the intron 9 (Simons et al. 2006). The first pair has forward primer 5'GCA-GTA-GCA-CCT-CAG-CAG-AGT and reverse primer 5'TTC-AGT-AAG-CTG-GTG-GAG-CA; the second pair has forward primer 5'AG-CTC-ATC-TTC-GTC-CCAGTG and reverse primer 5'TTC-AGT-AAG-CTG-GT G-GAG-CA.

\section{AFLP marker analysis}

For the AFLP marker analysis (Vos et al. 1995), restriction digestion using MseI and Sse8387I restriction enzymes, adapter ligation, PCR amplification and gel electrophoresis were carried out as described by Hartl et al. (1999) and Buerstmayr et al. (2002). Altogether, 64 AFLP primer combinations with two or three selective nucleotides on the $3^{\prime}$ end of either primer were performed for selective amplification. The $5^{\prime}$ end of the selective Sse8837I-primer was labelled with IRD700 or IRD800 when detected on an LI-COR 4200 dual-dye DNA analyser (LI-COR Biosciences, Lincoln, Nebraska USA), or was labelled with $C y 5$, $C y 3$ or FAM when scanned on a Typhoon-TRIO fluorescence scanner (GE Healthcare). AFLP markers were named according to the standard list for AFLP primer nomenclature (http://wheat.pw.usda.gov/ggpages/keygene AFLPs.html) followed by a number allocated to each polymorphic AFLP locus in this population within each primer combination, from smaller to larger fragments.

Statistical analysis

\section{Field data}

As a measure of FHB severity, the field scores were used to calculate the area under disease progress curve (AUDPC) as described by Buerstmayr et al. (2000). Analysis of variance (ANOVA) was calculated using the general linear model (GLM) procedure to estimate the effects of replications within experiments, experiments, genotypes and genotype-by-experiment interactions, with all effects treated as fixed. For the estimation of variance components, all effects were considered random. Broad-sense heritability was estimated from variance components with the equation $H^{2}=\sigma_{\mathrm{G}}^{2} /\left(\sigma_{\mathrm{G}}^{2}+\sigma_{\mathrm{G} \times \mathrm{E}}^{2} / e+\sigma_{\varepsilon}^{2} / e n\right)$, where $\sigma_{\mathrm{G}}^{2}=$ genotypic variance, $\quad \sigma_{\mathrm{G} \times \mathrm{E}}^{2}=$ genotype-by-experiment interaction variance, $\sigma_{\varepsilon}^{2}=$ error variance, $e=$ number of experiments, and $n=$ number of replications (Nyquist 1991). Pearson correlation coefficients were calculated for each experiment and trait combination. Statistical analyses were calculated in SAS/STAT version 9.2 (SAS Institute Inc 2008).

\section{Marker data}

Maps were calculated using CarthaGène version 0.999LKH for Linux (de Givry et al. 2005). We used a modified version of CarthaGène (provided by Dr. Clare Nelson, Kansas State University, USA) that allows map calculation in advanced backcross designs. For grouping a maximum distance of 30 centimorgan (cM) and a logarithm of odds (LOD) threshold of 3 were set. Linkage groups composed of many markers and those containing SSR markers with known map location on different chromosomes were grouped at higher LOD values. Cosegregating markers were merged into single markers. The most likely positions of the markers along the linkage groups were determined with the commands nicemapl, nicemapd, mfmapl, mfmapd, flips, build and annealing. The obtained maps were compared to the wheat consensus SSR map (Somers et al. 2004) available in the GrainGenes database (http://wheat. pw.usda.gov/ggpages/maps.shtml).

\section{QTL analysis}

QTL analysis was done with QGene (version 4.2.3) (Nelson 1997). The association between trait data and maker data was calculated by single-marker regression (SMR) and the locations of the detected QTL were estimated using simple interval mapping (SIM) (Haley and Knott 1992). The percentage of phenotypic variance (\%PV) explained by a QTL and its additive effect (add) were calculated. The critical LOD values at a type I error rate of $\alpha<0.05$ and $\alpha<0.01$ were determined by 1,000 permutations for each trait. Possible segregation distortion was tested using a Chi-square test for each marker. To estimate the total percentage of phenotypic variance explained by QTL, we fitted a linear model of all significant QTL simultaneously using the GLM procedure of SAS/STAT (SAS Institute Inc 2008). Linkage groups and LOD profiles were drawn with MapChart 2.2 (Voorrips 2002). 


\section{Results}

Trait variation and trait correlations

Means of the parents and the population, population range, least significant differences and broad-sense heritability for FHB severity and for several morphological traits are presented in Table 1. The population displayed significant variation for FHB severity, date of anthesis, plant height, spike length, number of spikelets per spike, density of the spikes, percent free threshing grains, glaucousness of spikes and leaf chlorosis. The population showed continuous variation for AUDPC means across all experiments. T. macha was the most resistant line on average over all experiments, Furore was susceptible, but several lines showed higher disease severity than Furore (Fig. 1).

Table 1 Means of parents and population, minimum and maximum scores of the population, least significant difference at $\alpha<0.05$ (LSD) and broad-sense heritability $\left(H^{2}\right)$ for FHB severity (AUDPC) and further plant traits

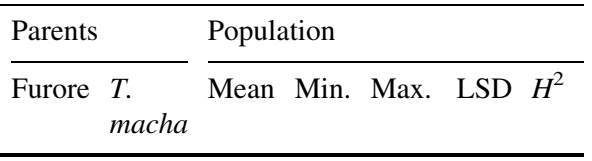

FHB severity (AUDPC)

\begin{tabular}{|c|c|c|c|c|c|c|c|}
\hline $\begin{array}{l}\text { Mean over all } \\
\text { experiments }\end{array}$ & 558 & 70 & 390 & 78 & 847 & 98 & \\
\hline Mean F. culm. & 946 & 114 & 639 & 163 & 1143 & 126 & \\
\hline Mean $F$. gram. & 267 & 32 & 207 & 32 & 639 & 71 & \\
\hline FG05 & 210 & 12 & 118 & 14 & 519 & 48 & \\
\hline FG06 & 392 & $-{ }^{\mathrm{f}}$ & 332 & 38 & 919 & 82 & \\
\hline FC06 & 1224 & $-{ }^{f}$ & 829 & 172 & 1395 & 131 & \\
\hline FG07 & 261 & 6 & 153 & 3 & 582 & 52 & \\
\hline $\mathrm{FC} 07$ & 860 & 96 & 535 & 64 & 1313 & 115 & \\
\hline FG08 & 206 & 64 & 216 & 33 & 771 & 88 & \\
\hline FC08 & 755 & 133 & 563 & 91 & 1272 & 132 & \\
\hline Date of anthesis ${ }^{\mathrm{a}}$ & 32.4 & 34.2 & 32.2 & 29.0 & 35.9 & 0.8 & \\
\hline Plant height $(\mathrm{cm})$ & 96.0 & 123.0 & 99.8 & 77.5 & 120.0 & 3.7 & \\
\hline Spike length $(\mathrm{cm})$ & 8.3 & 8.9 & 8.0 & 5.8 & 12.5 & 0.6 & \\
\hline Spikelets per spike & 15.2 & 18.6 & 15.7 & 12.6 & 20.4 & 1.1 & \\
\hline Spike density ${ }^{\mathrm{b}}$ & 1.8 & 2.1 & 2.0 & 1.4 & 2.7 & 0.2 & \\
\hline Threshability $(\%)^{\mathrm{c}}$ & 100 & 19.0 & 96.1 & 56.0 & 100 & 2.3 & \\
\hline $\begin{array}{l}\text { Glaucousness of } \\
\text { spikes }^{\mathrm{d}}\end{array}$ & 5.0 & 9.0 & 6.0 & 3.1 & 9.0 & 0.6 & \\
\hline Leaf chlorosis ${ }^{\mathrm{e}}$ & 0 & 0.15 & 1.4 & 0.2 & 8.0 & 0.5 & \\
\hline
\end{tabular}

a Number of days from 1 May to mid-anthesis

b Number of spikelets per $\mathrm{cm}$

${ }^{c}$ Percent free threshing seeds

${ }^{\mathrm{d}}$ Visually scored $0=$ non-glaucous to $9=$ extremely glaucous

e Visually scored $0=$ non-chlorotic to $9=$ extremely chlorotic

${ }^{\mathrm{f}}$ Missing values due to frost damage
Analysis of variance for AUDPC resulted in highly significant effects for all sources of variance (Table 2). The mean squares for genotypes were much larger than those for the genotype-by-experiment interaction, resulting in high broad-sense heritability of $H^{2}=0.88$ for AUDPC.

Pearson correlation coefficients for AUDPC between experiments were all positive and highly significant $(P<0.001)$ and ranged from $r=0.54$ to $r=0.76$. Inoculation with $F$. culmorum led to significantly higher average FHB severity than inoculation with $F$. graminearum (Table 1). The correlation between the average AUDPC obtained after inoculation with $F$. graminearum and $F$. culmorum was high $(r=0.82, P<0.001)$ and the correlations for the grain spawn-inoculated experiment (FG08) with $F$. graminearum and $F$. culmorum spray inoculated experiments ranged from $r=0.54$ to $r=0.62$.

Date of anthesis, spike density and threshability were positively correlated with FHB severity (AUDPC) and there was a negative correlation between FHB severity and plant height, spike length and glaucousness of the spikes (Table 3). Increased plant height, early flowering, long and lax spikes, and more intense spike glaucousness were associated with increased resistance to FHB. Plant height was associated with spike length, spike density,

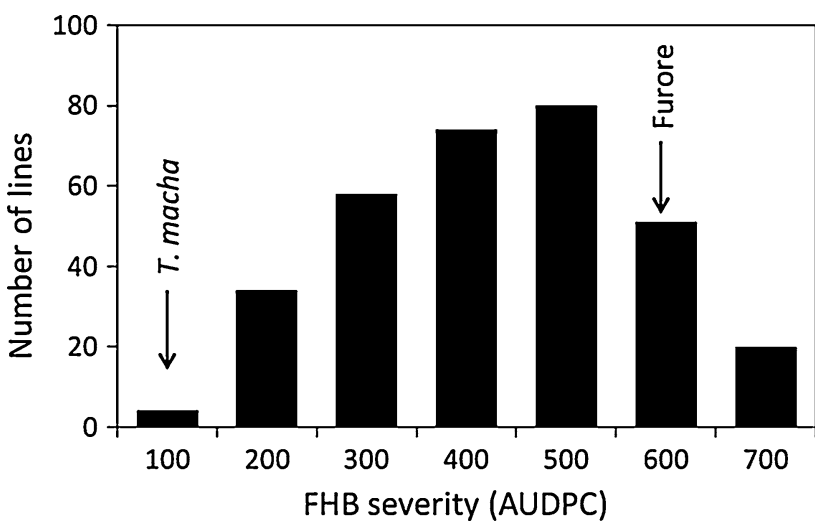

Fig. 1 Frequency distribution of $321 \mathrm{BC}_{2} \mathrm{~F}_{3}$ lines for FHB severity (AUDPC) means over all experiments. Arrows indicate the values of the parental lines

Table 2 Analysis of variance for FHB severity measured by AUDPC across seven experiments

\begin{tabular}{lrrrr}
\hline Source & DF & Mean square & $F$ value & $P$ value \\
\hline $\begin{array}{l}\text { Replication within } \\
\quad \text { experiment }\end{array}$ & 7 & $308,875.5$ & 24.5 & $<0.0001$ \\
Experiment & 6 & $41,005,134.6$ & 3257.5 & $<0.0001$ \\
Genotype & 322 & $273,034.1$ & 21.7 & $<0.0001$ \\
Genotype x experiment & 1916 & $34,100.3$ & 2.7 & $<0.0001$ \\
Error & 2178 & $12,587.8$ & & \\
\hline
\end{tabular}


Table 3 Pearson correlation coefficients among line mean values for AUDPC, date of anthesis, plant height, spike length, spikelet per spike, spike density, threshability and glaucousness of spikes

\begin{tabular}{|c|c|c|c|c|c|c|c|}
\hline Trait & Date of anthesis & Plant height & Spike length & Spikelets per spike & Spike density & Threshability & Glaucousness \\
\hline AUDPC & $0.30 * * *$ & $-0.53 * * *$ & $-0.27 * * *$ & 0.04 & $0.28 * * *$ & $0.44 * * *$ & $-0.19 * * *$ \\
\hline Date of anthesis & & -0.07 & 0.06 & $0.11 *$ & 0.00 & $0.23 * * *$ & 0.03 \\
\hline Plant height & & & $0.29 * * *$ & -0.05 & $-0.32 * * *$ & $-0.28 * * *$ & $0.21 * * *$ \\
\hline Spike length & & & & $0.39 * * *$ & $-0.75 * * *$ & $-0.35 * * *$ & $0.23 * * *$ \\
\hline Spikelets per spike & & & & & $0.29 * * *$ & 0.10 & $-0.17 * * *$ \\
\hline Spike density & & & & & & $0.38 * * *$ & $-0.36 * * *$ \\
\hline Threshability & & & & & & & $-0.30 * * *$ \\
\hline
\end{tabular}

$* P<0.05, * * P<0.01, * * * P<0.001$

glaucousness and threshability. Taller plants tended towards elongated, lax and more glaucous spikes, and had reduced threshability. The number of spikelets per spike was positively correlated with both spike length and density of the spikes. More compact types were non-glaucous and had free threshing seeds. Severity of leaf chlorosis was weakly correlated with plant height and not correlated with any other trait (results not shown).

Marker segregation and polymorphism

Among 282 SSR markers tested on the parents, 202 $(=72 \%)$ were polymorphic. Marker data generated on 300 lines with 128 SSR primer and 64 selective AFLP primer combinations and one morphological marker (hairy glumes) were used for map construction, with the SSR markers contributing 163 polymorphic loci and the AFLP analysis 533 clearly informative AFLP markers. After merging co-segregating markers into single markers, the remaining 560 unique markers were composed of 117 codominant and 443 dominant markers, among which T. macha carried the dominant allele for 230 and Furore for 213. The observed segregation in the population fitted the expected segregation ratio for a $\mathrm{BC}_{2} \mathrm{~F}_{3}$ RIL population. Only $33 \mathrm{cM}$ on parts of chromosomes 1D, 3A, 3D, 5A and one unassigned linkage group, and $62 \mathrm{cM}$ on parts of chromosomes 3A, 5A, 5B and three unlinked groups exhibited segregation distortion towards T. macha and Furore, respectively.

\section{Linkage map}

Of the 560 markers, 554 could be placed in 33 different linkage groups. The groups were assigned to individual wheat chromosomes using previously mapped SSR markers and their map information from GrainGenes as anchor points. Ten groups were assigned to the A genome, nine to the $\mathrm{B}$ genome and six to the $\mathrm{D}$ genome, while eight linkage groups that contained only AFLP markers remained unassigned. At least partial maps were obtained for all wheat chromosomes except $4 \mathrm{D}$. The total length of the map was $2,226 \mathrm{cM}$, with 803,830 and $455 \mathrm{cM}$ assigned, respectively, to the $\mathrm{A}, \mathrm{B}$ and $\mathrm{D}$ genomes and $138 \mathrm{cM}$ unassigned to specific chromosomes. The average distance between markers was $4 \mathrm{cM}$. The arrangement of common SSR markers were in most cases consistent with those in published maps in GrainGenes.

QTL analysis

\section{Quantitative trait loci mapping of FHB resistance}

Simple interval mapping identified five significant QTL on four different chromosomes associated with FHB severity measured by overall mean AUDPC across all experiments (Table 4). Permutation analysis resulted in LOD thresholds for AUDPC means of 3.8 and 4.2 at $\alpha<0.05$ and $\alpha<0.01$, respectively. For all of these five QTL, the allele improving resistance was derived from T. macha. The linkage groups and the LOD profiles for AUDPC means are shown in Fig. 2.

The QTL with the largest effect, explaining $23 \%$ of the phenotypic variance (PV) for AUDPC means mapped to chromosome 5AL at the interval between $X s 20 m 18 \_11$ and Xbarc 319 and peaked at the $Q$ locus; $11.5 \%$ of PV was explained by a QTL at the position of Xs11m24_10 on chromosome 2A. A QTL on the distal end of chromosome 2BL mapped close to Xwmc317 and accounted for $9.7 \%$ of the PV. The QTL on 2A was consistently found in all experiments with LOD values $>3$, while QTL on $5 \mathrm{AL}$ and 2BL were significant in six experiments. A second QTL on $2 \mathrm{~B}$ and one on $5 \mathrm{~B}$ were found in three and four experiments accounting for 7.1 and $9.3 \%$ of PV, respectively. On chromosome 2D, a QTL with LOD $>3$ was found in the $F$. graminearum-inoculated experiments only. The LOD value for means over all experiments was below significance. For this putative 2D QTL, the favourable allele was derived from Furore (Table 4; Fig. 2). The five significant 


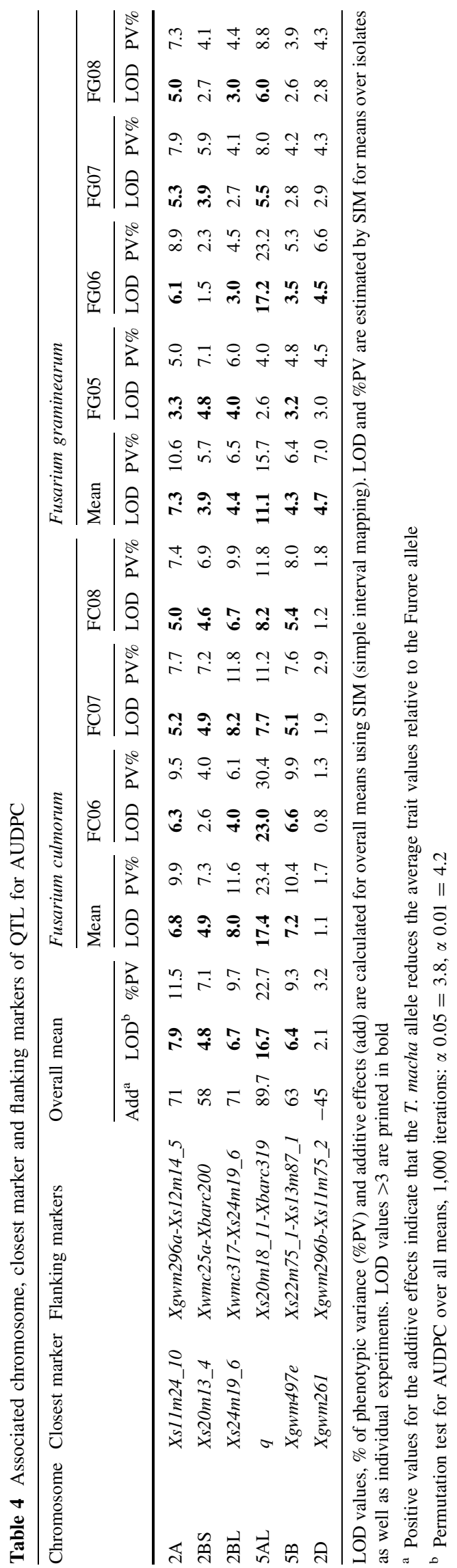

QTL together explained $48 \%$ of the phenotypic variance for mean AUDPC across all experiments.

\section{Quantitative trait loci of other traits}

The QTL identified for the date of anthesis, plant height, spike length, number of spikelets per spike, density of the spike, threshability, glaucousness of the spikes and leaf chlorosis are summarized in Table 5. Date of anthesis was modulated by QTL on 1B, 4A, 5AS, 5AL, 7B and 7D. The alleles from $T$. macha on chromosome 1B, 4A, 5AS, 5AL and $7 \mathrm{~B}$ accelerated flowering, and on 7D retarded flowering. Plant height was affected by QTL on chromosomes 2BL, 2D, 4A, 4B, 5AS, 5AL and 6A where the T. macha allele increased plant height, except for $2 \mathrm{D}$. A QTL for spike length was found on 5AL, with the T. macha allele favouring longer spikes. QTL affecting number of spikelets per spike were identified on 2A, 3B and 7A. QTL on 2D, 3B, 3D and 5AL influenced spike density. The T. macha allele at the 2D, 3B and 3D QTL increased density and at the 5AL QTL, laxness. Threshability was strongly associated with the $Q$ locus on 5AL. Glaucousness of the spike was influenced by QTL on chromosomes 1A, 2B and 5AL. Leaf chlorosis was influenced by one QTL detected on an unassigned group, with the T. macha allele conferring increased severity.

\section{FHB QTL and their association with QTL for other traits}

The large-effect QTL for AUDPC on 5AL was associated with several further traits: plant height, spike length, spike density, threshability and glaucousness of the spikes. Plants carrying the $T$. macha allele at this location were more resistant to FHB, taller and showed elongated lax, and more glaucous spikes and strongly reduced threshability. Co-localization of QTL for AUDPC and plant height was found on chromosome 2BL near Xwmc317, with the T. macha allele increasing FHB resistance and plant height. On chromosome 2D, QTL associated with plant height and spike density mapped to the same region, with the T. macha allele reducing plant height and favoring a dense spike. In terms of FHB, only $F$. graminearum-inoculated experiments with general low infection levels revealed a QTL with LOD as high as 3 at this locus.

\section{Discussion}

Precise phenotyping combined with a dense and reliable linkage map are essential for effective QTL detection. To ensure a complete and unbiased coverage of the donor genome, each of the $321 \mathrm{BC}_{2} \mathrm{~F}_{3: 4}$ lines was derived from a different $\mathrm{BC}_{1}$ plant. In other studies on wheat with the 


\section{Chromosome 2A}

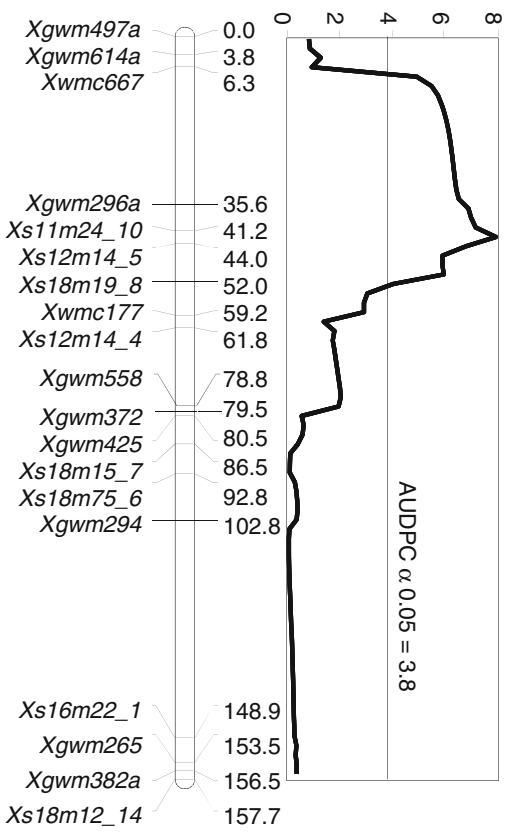

Chromosome 5A

\section{Chromosome 2B}

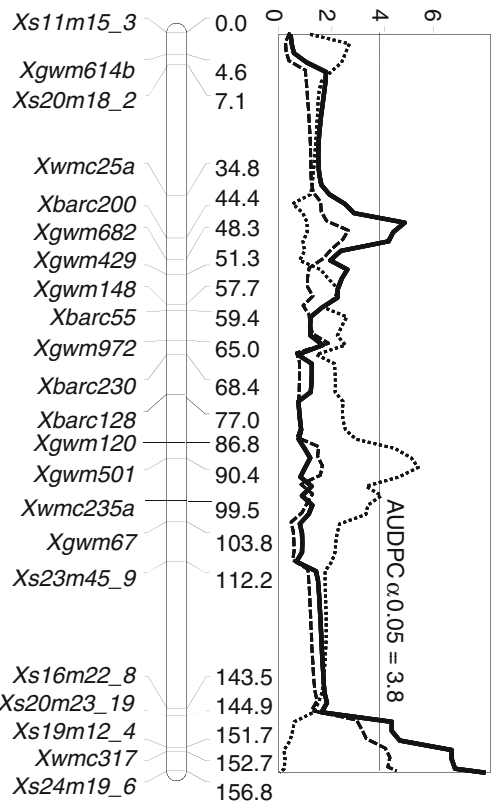

Chromosome2D

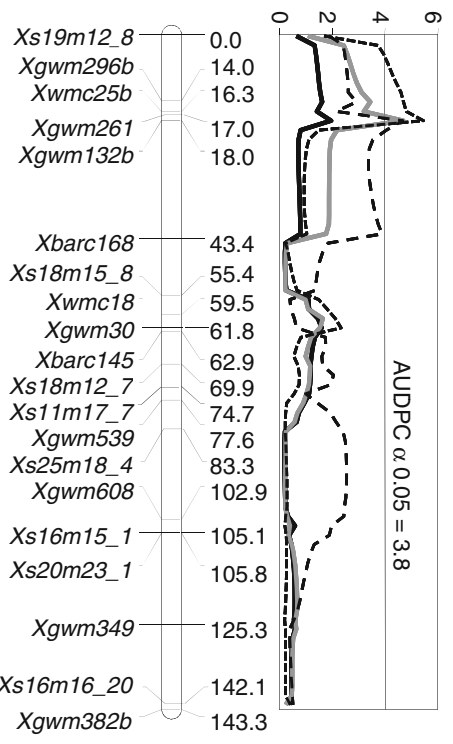

Chromosome 5B

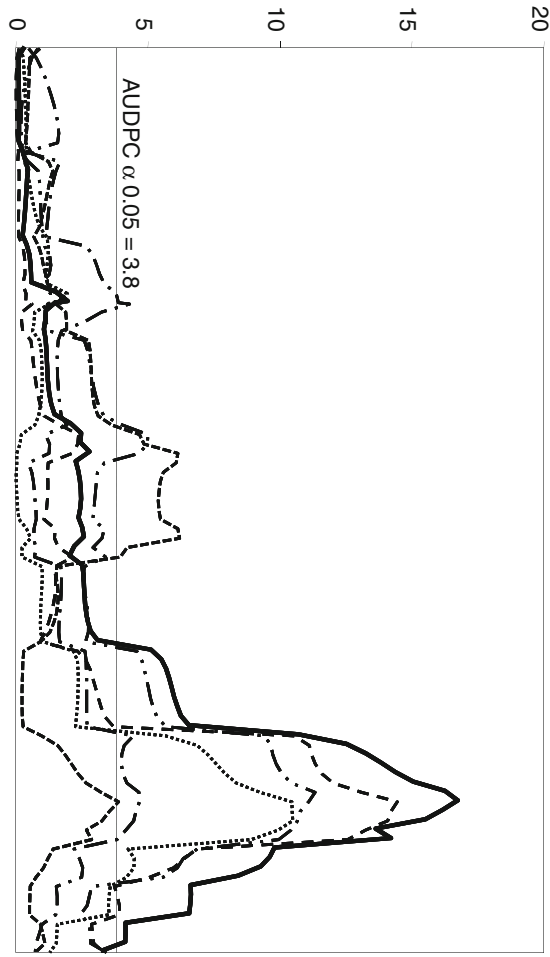

Fig. 2 Linkage maps and LOD profiles by SIM (simple interval mapping) for AUDPC means over all experiments on chromosome 2A, 2B, 2D, 5A, 5B and coinciding QTL for other traits

AB-QTL strategy, the populations were based on only few $\mathrm{BC}_{1}$ parents. Huang et al. (2004), Narasimhamoorthy et al. (2006), Liu et al. (2006), Leonova et al. (2007), Kunert et al. (2007), and Naz et al. (2008) produced populations descending from 42, 29, 15, 20, 42 and $22 \mathrm{BC}_{1}$ plants, which led to small recombination abilities resulting in low position resolution and spurious linkages (Narasimhamoorthy et al. 2006). By descending many $\mathrm{BC}_{2}$ lines each 
Table 5 Summary of QTL for morphological and developmental plant traits detected with SIM (simple interval mapping) and SMR (singlemarker regression). Significance thresholds are shown as footnotes

\begin{tabular}{|c|c|c|c|c|c|c|c|c|c|}
\hline \multirow[t]{2}{*}{ Trait } & \multirow[t]{2}{*}{ Chromosome } & \multirow[t]{2}{*}{ Closest marker } & \multirow[t]{2}{*}{ Flanking markers } & \multicolumn{3}{|l|}{ SIM } & \multicolumn{3}{|l|}{ SMR } \\
\hline & & & & $\operatorname{Add}^{\mathrm{i}}$ & LOD & $\% \mathrm{PV}$ & $\operatorname{Add}^{\mathrm{i}}$ & LOD & $\% \mathrm{PV}$ \\
\hline \multirow[t]{6}{*}{ Date of anthesis ${ }^{a}$} & 1B & $X s 23 m 13 \_8 a$ & $X s 23 m 13 \_8 a-X s 20 m 13 \_1$ & 0.4 & 5.6 & 8 & 0.4 & 5.6 & 8 \\
\hline & $4 \mathrm{~A}$ & $X s 14 m 22 \_6$ & Xs14m22_6-Xs14m87_5 & 0.5 & 12.0 & 17 & 0.6 & 11.1 & 16 \\
\hline & $5 \mathrm{AS}$ & Xbarc180 & Xbarc117-Xgwm156 & 0.2 & 3.9 & 6 & 0.3 & 4.3 & 6 \\
\hline & $5 \mathrm{AL}$ & $q$ & Xbarc319-Xs20m18_11 & 0.4 & 4.7 & 7 & 0.5 & 5.8 & 9 \\
\hline & $7 \mathrm{~B}$ & Xs19m19_11 & Xgwm611-Xs11m14_7 & 0.4 & 4.3 & 6 & 0.4 & 4.5 & 7 \\
\hline & $7 \mathrm{D}$ & Xgwm44 & Xs13m15_1-Xgwm111a & -0.4 & 3.6 & 5 & -0.5 & 5.4 & 8 \\
\hline \multirow[t]{7}{*}{ Plant height $^{\mathrm{b}}$} & $2 \mathrm{BL}$ & $X s 24 m 19 \_6$ & Xs19m12_4-Xs24m19_6 & -2.8 & 4.2 & 6 & -2.9 & 4.5 & 7 \\
\hline & $2 \mathrm{D}$ & Xgwm132b & Xgwm296b-Xs11m75_2 & 3.6 & 5.3 & 8 & 3.6 & 5.3 & 8 \\
\hline & $4 \mathrm{~A}$ & Xs14m13_9 & Xs18m24_2-Xgwm610 & -2.4 & 4.1 & 6 & -2.4 & 4.4 & 7 \\
\hline & 4B & $X s 18 m 12 \_6$ & $X s 23 m 45 \_12-X s 19 m 12 \_17$ & -2.7 & 4.0 & 6 & -2.7 & 4.3 & 6 \\
\hline & $5 \mathrm{AS}$ & Xgwm617 & Xgwm639-Xbarc151 & -2.9 & 6.1 & 9 & -3.0 & 6.3 & 9 \\
\hline & $5 \mathrm{AL}$ & $q$ & Xbarc319-Xs20m18_11 & -2.0 & 3.9 & 6 & -2.4 & 5.0 & 7 \\
\hline & $6 \mathrm{~A}$ & Xs19m19_3 & Xs18m24_8-Xbarc3 & -2.0 & 3.7 & 6 & -2.4 & 3.9 & 6 \\
\hline Spike length $^{\mathrm{c}}$ & $5 \mathrm{AL}$ & $q$ & Xbarc319-Xs20m18_11 & -0.6 & 11.3 & 16 & -0.6 & 12.4 & 17 \\
\hline \multirow[t]{3}{*}{ Spikelets per spike $^{\mathrm{d}}$} & $2 \mathrm{~A}$ & Xgwm425 & Xgwm588-Xs18m15_7 & -0.5 & 5.1 & 8 & -0.5 & 5.6 & 8 \\
\hline & $3 \mathrm{~B}$ & $X s 20 m 23 \_8$ & $X s 20 m 23 \_8-X s 22 m 12 \_1$ & -0.4 & 4.7 & 7 & -0.6 & 5.6 & 8 \\
\hline & $7 \mathrm{~A}$ & Xs13m17_6 & Xs11m75_6-Xs13m17_6 & 0.5 & 5.4 & 8 & 0.5 & 5.6 & 8 \\
\hline \multirow[t]{4}{*}{ Spike density ${ }^{\mathrm{e}}$} & $2 \mathrm{D}$ & Xgwm132b & Xgwm296b-Xbarc168 & -0.1 & 4.5 & 7 & -0.1 & 4.5 & 7 \\
\hline & $3 \mathrm{~B}$ & Xs11m19_9 & Xs14m12_4-Xbarc858 & -0.1 & 7.8 & 11 & -0.1 & 8.1 & 12 \\
\hline & $3 \mathrm{D}$ & Xgwm341 & Xs11m87_5-Xgwm341 & -0.1 & 5.6 & 8 & -0.1 & 5.7 & 8 \\
\hline & $5 \mathrm{AL}$ & $q$ & Xbarc319-Xs20m18_11 & 0.1 & 14.4 & 20 & 0.2 & 15.8 & 22 \\
\hline Threshability $^{\mathrm{f}}$ & $5 \mathrm{AL}$ & $q$ & Xbarc319-Xs20m18_11 & 10.0 & 64.8 & 63 & 10.8 & 81.2 & 71 \\
\hline \multirow[t]{3}{*}{ Glaucousness $^{\mathrm{g}}$} & $1 \mathrm{~A}$ & hairy glume & hairy glume-Xs20m15_8 & -0.7 & 12.1 & 17 & -0.7 & 12.1 & 17 \\
\hline & $2 \mathrm{~B}$ & Xgwm501 & Xs13m15_2-Xs19m19_14 & -0.5 & 5.3 & 8 & -0.6 & 5.7 & 8 \\
\hline & $5 \mathrm{AL}$ & $q$ & Xbarc319-Xs20m18_11 & -0.7 & 10.5 & 15 & -0.8 & 12.9 & 18 \\
\hline Leaf chlorosis ${ }^{\mathrm{h}}$ & $\mathrm{ni}^{\mathrm{j}}$ & Xs20m18_15 & Xbarc57b-Xs11m87_10 & -1.7 & 27.7 & 36 & -1.0 & 25.6 & 33 \\
\hline
\end{tabular}

${ }^{\mathrm{a}} \alpha 0.05=4, \alpha 0.01=5.2$

${ }^{\mathrm{b}} \alpha 0.05=3.8, \alpha 0.01=4.6$

c $\alpha 0.05=5.6, \alpha 0.01=7.5$

${ }^{\mathrm{d}} \alpha 0.05=4.4, \alpha 0.01=5.4$

e $\alpha 0.05=4.1, \alpha 0.01=5.1$

${ }^{\mathrm{f}} \alpha 0.05=6.6, \alpha 0.01=9$

$\mathrm{g} \alpha 0.05=3.9, \alpha 0.01=4.9$

${ }^{\mathrm{h}} \alpha 0.05=5.9, \alpha 0.01=7.3$

${ }^{\mathrm{i}}$ Positive values for the additive effects indicate that the T. macha allele reduces the average trait values relative to the Furore allele

${ }^{\mathrm{j}}$ Linkage group not assigned to a chromosome

from a different $\mathrm{BC}_{1}$ plant, a possible loss of parts of the donor genome was avoided. By using a large and unbiased population of $300 \mathrm{BC}_{2}$-derived lines, it was feasible to perform genetic mapping in the same population that was used for phenotyping and QTL mapping and the obtained maps appeared accurate in relation to other published maps.

We used spray inoculation and grain spawn inoculation methods for our experiments. As both methods provide information on overall FHB resistance, including type I (resistance to initial infection) and type II (resistance to the fungal spread) (Schroeder and Christensen 1963), the different types of resistance could not be separated. The significant and positive correlation coefficients among experiments and the high broad-sense heritability coefficient $\left(H^{2}=0.88\right)$ for FHB severity show that a large proportion of the observed variation among the tested lines was due to genetic effects and support the meaningful localization and estimation of QTL effects. 


\section{QTL detection}

Essentially the same QTL were found for experiments inoculated with $F$. graminearum as for those with $F$. culmorum, but for F. culmorum-inoculated experiments QTL were estimated more precisely and at higher probability values. This indicates that QTL detection is more effective under high infection pressure.

The detection of five putative QTL for FHB resistance underlines the complex and polygenic inheritance of this trait already shown in numerous other QTL projects for FHB in wheat. While Steed et al. (2005) reported a significant type 1 FHB-resistance QTL derived from $T$. macha, no 4A QTL was found in our study. Possibly, this 4A QTL was specific to their population based on a Hobbit 'sib' (T. macha 4A) single chromosome recombinant doubled haploid $(\mathrm{DH})$ population.

The largest-effect QTL in our study was found on chromosome 5AL at the $Q$ locus, where no QTL for FHB was reported previously. We consider it a major QTL for FHB resistance. It coincided with QTL associated with spike-related traits as for threshability, spike length, spike density, glaucousness of the spikes, date of anthesis and with plant height. Grausgruber et al. (1998) reported similar findings for morphological characters of the ear and found positive effects on resistance to $\mathrm{FHB}$ on $5 \mathrm{~A}$ in a Hobbit 'sib' (T. macha) intervarietal substitution line. T. macha carries the $q$ allele (Faris et al. 2005; Kuckuck 1964; Mac Key 1966; Simons et al. 2006) and the detected QTL very likely corresponds to $q$. The $Q$ locus with the alleles $Q$ (free threshing) and $q$ (speltoid, nonfree threshing) is a major regulatory gene. It pleiotropically influences many other domestication-related traits such as glume shape and tenacity, rachis fragility, spike length, plant height and spike emergence time (Faris and Gill 2002; Simons et al. 2006), which is in accordance with our findings. Whether the association with FHB resistance is due to a pleiotropic effect of the $q$ allele or close linkage between $q$ and a FHB-resistance QTL cannot be determined with current results. A much larger high-resolution mapping population would be needed to search for potential recombinants. Goral et al. (2008) tested several spelt wheat cultivars and found highly resistant as well as highly susceptible spelt varieties, and among $151 \quad T$. dicoccoides accessions tested by Buerstmayr et al. (2003) most were susceptible to FHB. Thus, the presence of the allele $q$ does not always confer high FHB resistance, supporting the hypothesis that FHB resistance is rather linked to $q$ than pleiotropic. In our population, it is possible that part of the increased FHB resistance associated with the $q$ allele may be due to morphological and developmental traits such as spike structure and flowering behaviour.
The QTL on 2BL close to Xwmc317 was repeatedly detected in six experiments. This QTL coincided with a QTL for plant height, with the Furore allele reducing height. The Rht4 gene is located on chromosome 2BL close to Xwmc317 (Ellis et al. 2005). Whether or not the QTL for reduced height from Furore is caused by Rht4 is currently not known. Schmolke et al. (2005) reported a resistance QTL for FHB in the Dream/Lynx population after spray inoculation in a similar position, but found no association with plant height. In our study, a second QTL on 2BS close to Xbarc200 was revealed in four experiments. Gilsinger et al. (2005) detected a resistance QTL linked with Xbarc200 on 2BS in the cultivar Goldfield and found a relation to narrow flower opening associated with resistance at this QTL. The flower opening trait was not investigated in our population. In the review of FHBrelated QTL by Buerstmayr et al. (2009), QTL for FHB resistance are reported at several positions along chromosome 2B. Since T. macha is related neither to Dream nor to Goldfield, we suggest that both T. macha-derived QTL on $2 \mathrm{~B}$ are novel.

In the $2 \mathrm{AS}$ region containing a FHB-resistance QTL significant in all experiments, no overlapping QTL for other traits were found. Three QTL related to FHB resistance were published on $2 \mathrm{AS}$. The Asian wheat variety Ning7840 expressed a minor QTL for FHB spread associated with Xgwm614 (Zhou et al. 2002). Variety NK93604 had a QTL for low DON (deoxynivalenol) accumulation at Xbarc124, although no association to FHB symptom severity was found (Semagn et al. 2007), and a resistance QTL derived from Wangshuibai mapped close to Xgwm425 near the centromere. In the present population, Xgwm614a mapped $38 \mathrm{cM}$ distal and Xgwm425 $40 \mathrm{cM}$ proximal to our QTL peak at the AFLP marker Xs11m24_10. We therefore assume that the $T$. macha QTL differs from these previously reported QTL at chromosome 2A. Based on our map and the maps of Xue et al. (2008) and Song et al. (2005), it appears likely that QTL for FHB severity on 2AS, 2BS and $2 \mathrm{D}$ reside at homoeologous genomic regions.

Bourdoncle and Ohm (2003) reported a QTL for FHB spread derived from Patterson with minor effects towards resistance in approximately the same location as our 5BL QTL observed in five experiments.

Morphological traits and their association with FHB resistance

\section{$Q$ locus}

A T. macha line with good resistance to FHB served as donor parent. This line was $27 \mathrm{~cm}$ taller and 2 days later in anthesis compared to the recurrent parent Furore, a common hexaploid wheat. Ear morphology of T. macha differs 
markedly from the bread wheat parent. T. macha is a hulled wheat, has a dense square-headed, but non-compact spike phenotype, has a fragile rachis, is only partly free threshing and has hairy and waxy glumes. Compared to $T$. spelta it does not show the speltoid ear shape, which is described as a spear-shaped spike with elongated rachis. The European spelt and $T$. macha carry the $q$ allele, while the Iranian spelt carries $Q$ (Simons et al. 2006). The $q$ allele plays a key role in the present study. In accordance with the known modifications associated with the $q$ allele, we identified QTL for plant height, date of anthesis, spike density, spike length and threshability at the location of $q$. In addition, we found QTL for glaucousness of the glumes and for FHB severity. Lines carrying the $q$ allele showed typically speltoid earshape, which was not visible in T. macha, but appeared in the genetic background of Furore.

\section{Compactness}

T. macha has a compacting gene that appears to be allelic to $C$ (Swaminathan and Rao 1961). $C$ determines whether a spike is lax or compacted and it affects spike morphology, grain size, shape, spikelet number and perhaps other aspects of plant development (Gul and Allan 1972). In our study, QTL for plant height and dense spike co-located on chromosome 2D near Xgwm261. The T. macha allele changed lines towards smaller plants with dense squareheaded spikes. Additionally, a minor but not significant QTL for FHB resistance coincided. Lines carrying T. macha allele were more highly infected. Johnson et al. (2008) mapped $C$ to $X g w m 358$ close to the centromere of 2D, which is in disagreement with our findings. Sourdille et al. (2000) and Ma et al. (2007) found a QTL for compactness on 2D at Xgwm261, consistent with our results. Rht8 (Worland et al. 1998) is closely linked to Xgwm261, consistent with the QTL for plant height in our study. Nalam et al. (2007) and Ma et al. (2007) reported a QTL for glume tenacity and threshability at Xgwm261. The basically nonfree threshing character of $T$. macha in combination with its long and dense but non-compact spike arises therefore from the specific allele combination of $q$ and $C$.

A QTL for density of spikes was found at Xgwm341 on 3D. Xgwm341 maps about $0.2 \mathrm{cM}$ from $X g w m 456$ (Somers et al. 2004). The $S 1$ locus was mapped close to the centromeric marker Xgwm456 of the long arm of 3D by Salina et al. (2000). Rao (1977) and Koba and Tsunewaki (1978) also reported that the sphaerococcum gene $S 1$ is near the centromere of chromosome 3D. Besides other characteristics, $S 1$ also influences spike density. Swaminathan and Rao (1961) confirmed that the qq CC S1S1 alleles were present in T. macha. Even though the genotype of both T. macha and Furore are assumed to be S1S1, the T. macha allele conferred a denser spike than Furore. This indicates that T. macha and Furore carry different alleles at this locus. Interestingly, no QTL for number of spikelets per spike coincided with the previously discussed loci on 5AL, 2D and 3D.

\section{Glaucousness (waxiness/glossiness) of the spike}

QTL for spike glaucousness coincided with QTL for FHB resistance on chromosome 5AL and the morphological marker $\mathrm{Hg}$ (hairy glumes) on $1 \mathrm{AS}$. The QTL for glaucousness on $2 \mathrm{~B}$ had no association with other traits. Glaucousness could possibly be a passive resistance factor, as a more waxy cuticle can potentially impede the entry of fungi. The positive phenotypic correlation between glaucousness and resistance may be accounted for by coinciding QTL for glaucousness and QTL for FHB resistance on $5 \mathrm{AL}$ rather then by glaucousness per se.

\section{Plant height}

Although seven QTL with significant influence on plant height were revealed, only QTL on 5AL and 2BL coincided with QTL for FHB resistance. Repeated observations of associations between FHB severity and plant height (Buerstmayr et al. 2000; Gervais et al. 2003; Hilton et al. 1999; Mesterhazy 1995; Steiner et al. 2004) as well as compactness of the spikes (Steiner et al. 2004; Zhu et al. 1999) may be partly explained by passive resistance. Tall plants are more exposed to wind and sun and therefore dry off earlier, whereby short plants, especially in combination with dense spikes, are exposed to a more humid microclimate, which favours FHB infections.

\section{Date of flowering}

Although T. macha was the later-flowering parent, the T. macha allele at QTL for date of anthesis favoured earlier flowering on chromosomes 1B, 4A, 5AS, 5AL and 7B and delayed flowering only on 7D. The chromosome 4A QTL showed the highest influence on the date of anthesis. Though FHB resistance was positively correlated with date of anthesis, no coinciding QTL except the 5AL one was revealed. Several different publications mention a positive effect of early flowering on FHB resistance (Gervais et al. 2003; Holzapfel et al. 2008; Paillard et al. 2004; Schmolke et al. 2005; Steiner et al. 2004) in agreement with our findings.

\section{Chlorosis}

A single major QTL for leaf chlorosis was found on an unassigned linkage group. This is in agreement with Tsunewaki (1971) and Naskidashvili et al. (2010) who 
reported the occurrence of several chlorosis and necrosis alleles in T. macha. Leaf chlorosis was not associated with other traits evaluated in this study.

\section{Summary and conclusions}

In this AB-QTL analysis, several novel QTL deriving from the landrace $T$. macha were mapped. These novel $T$. $m a$ cha-derived QTL appear valuable for broadening the genetic basis and diversity of FHB resistance in wheat. Some of the FHB-resistance QTL from Georgian spelt wheat coincide with those for spelt wheat traits. The major FHB-resistance QTL at chromosome 5A was closely associated with the wild-type allele $q$, and it is unclear whether $q$ has a pleiotropic effect on FHB resistance or is closely linked to a nearby resistance QTL. Only the 2BL FHB-resistance QTL co-located with a plant height QTL; the allele $q$ had only a moderate effect on height and none of the other plant height QTL corresponded to FHBresistance QTL. Selected lines from this study appear useful for practical resistance breeding, because they carry QTL from the landrace T. macha in an already agronomically adapted background. In addition, selected lines carrying $q$ (spelt wheat ear type) could be useful as crossing partners for spelt wheat breeding.

Acknowledgments Funding of this project was provided by Austrian Science Fund (FWF), project number: 17310-B05. We acknowledge the Federal State of Lower Austria for supporting the research program of IFA-Tulln. We acknowledge the excellent technical assistance by Lisa Schmid and Matthias Fidesser. We thank Clare Nelson (Kansas State University, USA) for adapting the CarthaGène program to handle advanced backcross designs and for his suggestions for improving this manuscript.

Open Access This article is distributed under the terms of the Creative Commons Attribution Noncommercial License which permits any noncommercial use, distribution, and reproduction in any medium, provided the original author(s) and source are credited.

\section{References}

Barisashvili MA, Gorgidze AD (1979) O proiskhozhdenii pshenitsy T. macha Dek. et. Men. (On the origin of T. macha Dek. et Men. wheat) Soobshcheniya. Akad Nauk Gruz SSR 95:409-412

Bourdoncle W, Ohm HW (2003) Quantitative trait loci for resistance to Fusarium head blight in recombinant inbred wheat lines from the cross Huapei 57-2/Patterson. Euphytica 131:131-136

Buerstmayr H, Lemmens M, Grausgruber H, Ruckenbauer P (1996) Scab resistance of international wheat germplasm. Cereal Res Commun 24:195-202

Buerstmayr H, Steiner B, Lemmens M, Ruckenbauer P (2000) Resistance to fusarium head blight in winter wheat: heritability and trait associations. Crop Sci 40:1012-1018

Buerstmayr H, Lemmens M, Hartl L, Doldi L, Steiner B, Stierschneider M, Ruckenbauer P (2002) Molecular mapping of QTLs for
Fusarium head blight resistance in spring wheat. I. Resistance to fungal spread (type II resistance). Theor Appl Genet 104:84-91

Buerstmayr H, Stierschneider M, Steiner B, Lemmens M, Griesser M, Nevo E, Fahima T (2003) Variation for resistance to head blight caused by Fusarium graminearum in wild emmer (Triticum dicoccoides) originating from Israel. Euphytica 130:17-23

Buerstmayr H, Ban T, Anderson JA (2009) QTL mapping and marker-assisted selection for Fusarium head blight resistance in wheat: a review. Plant Breed 128:1-26

Cao WG, Scoles G, Hucl P, Chibbar RN (2000) Phylogenetic relationships of five morphological groups of hexaploid wheat (Triticum aestivum L. em Thell.) based on RAPD analysis. Genome 43:24-727

de Givry S, Bouchez M, Chabrier P, Milan D, Schiex T (2005) CARTHAGENE: multipopulation integrated genetic and radiated hybrid mapping. Bioinformatics 21:1703-1704

Ellis MH, Rebetzke GJ, Azanza F, Richards RA, Spielmeyer W (2005) Molecular mapping of gibberellin-responsive dwarfing genes in bread wheat. Theor Appl Genet 111:423-430

Faris JD, Gill BS (2002) Genomic targeting and high-resolution mapping of the domestication gene $Q$ in wheat. Genome 45:706-718

Faris JD, Simons KJ, Zhang Z, Gill BS (2005) The wheat super domestication gene $Q$. Wheat Inf Serv 100:129-148

Gervais L, Dedryver F, Morlais JY, Bodusseau V, Negre S, Bilous M, Groos C, Trottet M (2003) Mapping of quantitative trait loci for field resistance to Fusarium head blight in an European winter wheat. Theor Appl Genet 106:961-970

Gilsinger J, Kong L, Shen X, Ohm H (2005) DNA markers associated with low Fusarium head blight incidence and narrow flower opening in wheat. Theor Appl Genet 110:1218-1225

Goral T, Ochodzki P, Mazurek A, Bulinska-Radomska Z (2008) Resistance of species from genus Triticum to Fusarium head blight and accumulation of Fusarium-metabolites in grain. Cereal Res Commun 36:95-97

Grausgruber H, Lemmens M, Buerstmayr H, Ruckenbauer P (1998) Chromosomal location of Fusarium head blight resistance and in vitro toxin tolerance in what using the Hobbit 'sib' (Triticum macha) chromosome substitution lines. J Genet Breed 52:173-180

Gul A, Allan RE (1972) Relation of the club gene with yield and yield components of near-isogenic lines. Crop Sci 12:297

Haley SC, Knott AS (1992) A simple regression method for mapping quantitative trait loci in line crosses using flanking markers. Heredity 69:315-324

Hartl L, Mohler V, Zeller FJ, Hsam SLK, Schweizer G (1999) Identification of AFLP markers closely linked to the powdery mildew resistance genes $\mathrm{Pm} 1 \mathrm{c}$ and $\mathrm{Pm} 4 \mathrm{a}$ in common wheat (Triticum aestivum L.). Genome 42:322-329

Hilton AJ, Jenkinson P, Hollins TW, Parry DW (1999) Relationship between cultivar height and severity of Fusarium ear blight in wheat. Plant Pathol 48:202-208

Holzapfel J, Voss HH, Miedaner T, Korzun V, Haberle J, Schweizer G, Mohler V, Zimmermann G, Hartl L (2008) Inheritance of resistance to Fusarium head blight in three European winter wheat populations. Theor Appl Genet 117:1119-1128

Huang XQ, Kempf H, Ganal MW, Roder MS (2004) Advanced backcross QTL analysis in progenies derived from a cross between a German elite winter wheat variety and a synthetic wheat (Triticum aestivum L.). Theor Appl Genet 109:933-943

Johnson EB, Nalam VJ, Zemetra RS, Riera-Lizarazu O (2008) Mapping the compactum locus in wheat (Triticum aestivum L.) and its relationship to other spike morphology genes of the Triticeae. Euphytica 163:193-201

Koba T, Tsunewaki K (1978) Mapping of the $s$ and Ch2 genes on chromosome 3D of common wheat. Wheat Inf Serv 45-46:18-20 
Kuckuck H (1964) Experimentelle Untersuchungen zur Entstehung der Kulturweizen. Z Pflanzenzuchtg 51:97-140

Kunert A, Naz AA, Dedeck O, Pillen K, Leon J (2007) AB-QTL analysis in winter wheat: I. Synthetic hexaploid wheat (T. turgidum ssp dicoccoides $\times T$. tauschii) as a source of favourable alleles for milling and baking quality traits. Theor Appl Genet 115:683-695

Law CN, Worland AJ (1996) Inter-varietal chromosome substitution lines in wheat. Euphytica 89:1-10

Leonova IN, Laikova LI, Popova OM, Unger O, Borner A, Roder MS (2007) Detection of quantitative trait loci for leaf rust resistance in wheat-T. timopheevii/T. tauschii introgression lines. Euphytica 155:79-86

Liu SB, Zhou RG, Dong YC, Li P, Jia JZ (2006) Development, utilization of introgression lines using a synthetic wheat as donor. Theor Appl Genet 112:1360-1373

Liu SY, Hall MD, Griffey CA, McKendry AL (2009) Meta-analysis of QTL associated with fusarium head blight resistance in wheat. Crop Sci 49:1955-1968

Löffler M, Schön CC, Miedaner T (2009) Revealing the genetic architecture of FHB resistance in hexaploid wheat (Triticum aestivum L.) by QTL meta-analysis. Mol Breed 23:473-488

Ma ZQ, Zhao DM, Zhang CQ, Zhang ZZ, Xue SL, Lin F, Kong ZX, Tian DG, Luo QY (2007) Molecular genetic analysis of five spike-related traits in wheat using RIL and immortalized F-2 populations. Mol Genet Genomics 277:31-42

Mac Key J (1954) Neutron and X-ray experiments in wheat and a revision of the speltoid problem. Hereditas 40:65-180

Mac Key J (1966) Species relationship in Triticum. Hereditas 2(Suppl):237-276

Mentewab A, Rezanoor HN, Gosman N, Worland AJ, Nicholson P (2000) Chromosomal location of Fusarium head blight resistance genes and analysis of the relationship between resistance to head blight and brown foot rot. Plant Breed 119:15-20

Mesterhazy A (1995) Types and components of resistance to Fusarium head blight of wheat. Plant Breed 114:377-386

Morris R, Sears ER (1967) The cytogenetics of wheat and its relatives. In: Quisenberry KS, Reitz LP (eds) Wheat and wheat improvement. American Society of Agronomy, Madison, pp 19-87

Nalam VJ, Vales MI, Watson CJW, Johnson EB, Riera-Lizarazu O (2007) Map-based analysis of genetic loci on chromosome 2D that affect glume tenacity and threshability, components of the free-threshing habit in common wheat (Triticum aestivum L.). Theor Appl Genet 116:135-145

Narasimhamoorthy B, Gill BS, Fritz AK, Nelson JC, Brown-Guedira GL (2006) Advanced backcross QTL analysis of a hard winter wheat $\times$ synthetic wheat population. Theor Appl Genet 112:787-796

Naskidashvili P, Naskidashvili M, Naskidashvili I, Gakharia N (2010) Revealing genes of hybrid necrosis and red hybrid chlorosis in crosses of varieties of Georgian wheat and the importance of these genetic phenomena for selection and theoretical research. Bull Georg Natl Acad Sci 4:145-150

Naz AA, Kunert A, Lind V, Pillen K, Leon J (2008) AB-QTL analysis in winter wheat: II. Genetic analysis of seedling and field resistance against leaf rust in a wheat advanced backcross population. Theor Appl Genet 116:1095-1104

Nelson J (1997) QGENE: software for marker-based genomic analysis and breeding. Mol Breed 3:239-245

Nyquist WE (1991) Estimation of heritability and prediction of selection response in plant populations. Crit Rev Plant Sci 10:235-322

Paillard S, Schnurbusch T, Tiwari R, Messmer M, Winzeler M, Keller B, Schachermayr G (2004) QTL analysis of resistance to
Fusarium head blight in Swiss winter wheat (Triticum aestivum L.). Theor Appl Genet 109:323-332

Pestsova E, Ganal MW, Roder MS (2000) Isolation and mapping of microsatellite markers specific for the $\mathrm{D}$ genome of bread wheat. Genome 43:689-697

Rao MVP (1972) Mapping of the compactum gene $C$ on chromosome 2D of wheat. Wheat Inf Serv 35:9

Rao MVP (1977) Mapping of the sphaerococcum gene ' $s$ ' on chromosome 3D of wheat. Cereal Res Commun 5:15-17

Roeder SM, Korzun K, Wendehake K, Plaschke J, Tixier HM, Leroy P, Ganal WM (1998) A microsatellite map of wheat. Genetics 149:2007-2023

Saghai Maroof MAK, Soliman RA, Jorgensen RA, Allard RW (1984) Ribosomal DNA spacer length polymorphism in barley: Mendelian inheritance, chromosomal location and populationdynamics. Proc Natl Acad Sci USA 81:8014-8018

Salina E, Borner A, Leonova I, Korzun V, Laikova L, Maystrenko O, Roder MS (2000) Microsatellite mapping of the induced sphaerococcoid mutation genes in Triticum aestivum. Theor Appl Genet 100:686-689

SAS Institute Inc (2008) SAS/STAT ${ }^{\circledR} 9.2$ user's guide. Cary, NC

Schmolke M, Zimmermann G, Buerstmayr H, Schweizer G, Miedaner T, Korzun V, Ebmeyer E, Hartl L (2005) Molecular mapping of Fusarium head blight resistance in the winter wheat population Dream/Lynx. Theor Appl Genet 111:747-756

Schroeder HW, Christensen JJ (1963) Factors affecting resistance of wheat to scab caused by Gibberella zeae. Phytopathology $53: 831-838$

Semagn K, Skinnes H, Bjornstad A, Maroy AG, Tarkegne Y (2007) Quantitative trait loci controlling Fusarium head blight resistance and low deoxynivalenol content in hexaploid wheat population from 'Arina' and NK93604. Crop Sci 47:294-303

Simons KJ, Fellers JP, Trick HN, Zhang ZC, Tai YS, Gill BS, Faris JD (2006) Molecular characterization of the major wheat domestication gene $Q$. Genetics 172:547-555

Somers DJ, Isaac P, Edwards K (2004) A high-density microsatellite consensus map for bread wheat (Triticum aestivum L.). Theor Appl Genet 109:1105-1114

Song QJ, Shi JR, Singh S, Fickus EW, Costa JM, Lewis J, Gill BS, Ward R, Cregan PB (2005) Development and mapping of microsatellite (SSR) markers in wheat. Theor Appl Genet 110:550-560

Sourdille P, Tixier MH, Charmet G, Gay G, Cadalen T, Bernard S, Bernard M (2000) Location of genes involved in ear compactness in wheat (Triticum aestivum) by means of molecular markers. Mol Breed 6:247-255

Steed A, Chandler E, Thomsett M, Gosman N, Faure S, Nicholson P (2005) Identification of type I resistance to Fusarium head blight controlled by a major gene located on chromosome $4 \mathrm{~A}$ of Triticum macha. Theor Appl Genet 111:521-529

Steiner B, Lemmens M, Griesser M, Scholz U, Schondelmaier J, Buerstmayr H (2004) Molecular mapping of resistance to Fusarium head blight in the spring wheat cultivar Frontana. Theor Appl Genet 109:215-224

Swaminathan MS, Rao MVP (1961) Macro-mutations and subspecific differentiation in Triticum. Wheat Inf Serv 13:9-11

Tanksley SD, Nelson JC (1996) Advanced backcross QTL analysis: a method for the simultaneous discovery and transfer of valuable QTLs from unadapted germplasm into elite breeding lines. Theor Appl Genet 92:191-203

Tsunewaki K (1971) Distribution of necrosis genes in wheat v. Triticum macha, T. spelta and T. vavilovii. Jpn J Genet 46:93-101

Voorrips RE (2002) MapChart: software for the graphical presentation of linkage maps and QTLs. J Hered 93:77-78 
Vos P, Hogers R, Bleeker M, Reijans M, van de Lee T, Horens M, Frijters A, Pot J, Peleman J, Kuiper M, Zabeau M (1995) AFLP: a new technique for DNA fingerprinting. Nucleic Acids Res 23:4407-4414

Worland AJ, Korzun V, Roder MS, Ganal MW, Law CN (1998) Genetic analysis of the dwarfing gene Rht 8 in wheat. Part II. The distribution and adaptive significance of allelic variants at the $R h t 8$ locus of wheat as revealed by microsatellite screening. Theor Appl Genet 96:1110-1120

Xue SL, Zhang ZZ, Lin F, Kong ZX, Cao Y, Li CJ, Yi HY, Mei MF, Zhu HL, Wu J, Xu HB, Zhao DM, Tian DG, Zhang CQ, Ma Z (2008) A high-density intervarietal map of the wheat genome enriched with markers derived from expressed sequence tags. Theor Appl Genet 117:181-189

Zhou WC, Kolb FL, Bai GH, Shaner G, Domier LL (2002) Genetic analysis of scab resistance QTL in wheat with microsatellite and AFLP markers. Genome 45:719-727

Zhu H, Gilchrist L, Hayes P, Kleinhofs A, Kudrna D, Liu Z, Prom L, Steffenson B, Toojinda T, Vivar H (1999) Does function follow form? Principal QTLs for Fusarium head blight (FHB) resistance are coincident with QTLs for inflorescence traits and plant height in a doubled-haploid population of barley. Theor Appl Genet 99:1221-1232 